\title{
ON AN INTRIGUING INTEGRAL AND SOME SERIES RELATED TO $\zeta(4)$
}

\author{
DAVID BORWEIN AND JONATHAN M. BORWEIN
}

(Communicated by Andrew Bruckner)

\begin{abstract}
An intriguing log-cosine integral is fully analyzed and shown to have value a rational multiple of $\pi \zeta(4), \zeta$ being the Riemann zeta function. From this we deduce by means of generating functions and Parseval's identity the sums of certain series previously established by a completely different method.
\end{abstract}

\section{INTRODUCTION}

The intention of this note is to provide a complete proof of the following formula:

$$
\frac{1}{\pi} \int_{0}^{\pi} \theta^{2} \log ^{2}\left(2 \cos \frac{1}{2} \theta\right) d \theta=\frac{11 \pi^{4}}{180}=\frac{11}{2} \zeta(4) .
$$

From this we will derive, inter alia, the identity

$$
\sum_{n=1}^{\infty}\left(\frac{1+\frac{1}{2}+\cdots+\frac{1}{n}}{n}\right)^{2}=\frac{17 \pi^{4}}{360}=\frac{17}{4} \zeta(\cdot 4) .
$$

This identity was surprising and new to us when Enrico Au-Yeung (an undergraduate student in the Faculty of Mathematics in Waterloo) conjectured it on the basis of a computation of 500,000 terms (five digit accuracy!); our first impulse was to perform a higher-order computation to show it to be false. It is not easy to naively compute the value of the sum to more than about eight places.

It occurred to us that we might be able to (i) obtain a function whose Fourier sine series was

$$
\sum_{n=1}^{\infty} \frac{1}{n}\left(1+\frac{1}{2}+\cdots+\frac{1}{n}\right) \sin n t
$$

and (ii) numerically determine the value of the desired sum via Parseval's equation. This led to equation (12) below and an evaluation of the sum to 25 digits

Received by the editors July 20, 1993.

1991 Mathematics Subject Classification. Primary 42A24, 11M99; Secondary 33B15, 42A16, 65B10, 65B15.

Key words and phrases. Riemann zeta function, Parseval's identity, generating functions, logcosine integrals, polylogarithms.

This research was supported in part by the Natural Sciences and Engineering Research Council of Canada. 
in a couple of minutes in Maple on a work station. Now armed with the assurance that the result was true, we were prepared to look for the closed form evaluation presented below. We did not know at the time that P. J. De Doelder $[4,(9)]$ had established (2) below from which the above sum is an immediate consequence. In $\S 5$ below we present complete, self-contained and shorter versions of De Doelder's proofs of this and related sums.

We believe our derivations make a very pleasant tour through diverse topics in Classical Analysis, almost all of which are well described in [1] or [6]. We will use the following standard definitions and identities involving the Riemann zeta function $\zeta$, the beta function $\beta$, the gamma function $\Gamma$, and the digamma function $\psi$ :

$$
\begin{gathered}
\zeta(2)=\frac{\pi^{2}}{6}, \quad \zeta(4)=\frac{\pi^{4}}{90}, \quad \text { where } \quad \zeta(z):=\sum_{n=1}^{\infty} \frac{1}{n^{z}} \text { for } \operatorname{Re} z>1 \\
\beta(x, y):=\int_{0}^{1}(1-u)^{x-1} u^{y-1} d u=\frac{\Gamma(x) \Gamma(y)}{\Gamma(x+y)} \text { for } x>0, y>0
\end{gathered}
$$

(see [6, Theorem 7.69]);

$$
\Gamma(z) \Gamma(1-z) \sin \pi z=\pi
$$

(see [6, Theorem 7.64]);

$\psi(1+z):=\frac{d}{d z} \log \Gamma(1+z), \quad \psi^{\prime}(1+z)=\sum_{n=2}^{\infty}(-1)^{n}(n-1) \zeta(n) z^{n-2}$ for $|z|<1$ (see [6, Theorem 7.71]).

\section{THE MAIN RESULTS}

Theorem.

$$
\frac{1}{\pi} \int_{0}^{\pi} \theta^{2} \log ^{2}\left(2 \cos \frac{1}{2} \theta\right) d \theta=\frac{11 \pi^{4}}{180}=\frac{11}{2} \zeta(4) .
$$

Corollary. Let $H_{n}:=\sum_{k=1}^{n} \frac{1}{k}$. Then

$$
\begin{gathered}
\sum_{n=1}^{\infty} \frac{H_{n}^{2}}{(n+1)^{2}}=\frac{11 \pi^{4}}{360}=\frac{11}{4} \zeta(4), \\
\sum_{n=1}^{\infty} \frac{H_{n}^{2}}{n^{2}}=\frac{17 \pi^{4}}{360}=\frac{17}{4} \zeta(4),
\end{gathered}
$$

and

$$
\sum_{n=1}^{\infty} \frac{H_{n}}{n^{3}}=\frac{\pi^{4}}{72}=\frac{5}{4} \zeta(4)
$$

\section{Proofs of THE MAIN ResUlts}

Proof of the Theorem. Suppose initially that $x \geq 0, \eta \geq 0$. Integrating $(1+w)^{x} w^{\eta}$ in the $w=u+i v$ plane around the contour bounded by the $u$-axis from -1 to 1 and the upper semicircle of unit radius yields

$$
\int_{-1}^{1}(1+w)^{x} w^{\eta} d w=-i \int_{0}^{\pi}\left(1+e^{i \theta}\right)^{x} e^{i(\eta+1) \theta} d \theta
$$


since $(1+w)^{x} w^{\eta}$ is holomorphic within and continuous on and within the contour (see [3]). The imaginary part of the right-hand term is

$$
-\int_{0}^{\pi}\left(2 \cos \frac{1}{2} \theta\right)^{x} \cos y \theta d \theta
$$

with $y:=\eta+\frac{1}{2} x+1$, and the left-hand term equals

$$
\int_{0}^{1}(1+u)^{x} u^{\eta} d u+e^{i \pi \eta} \int_{0}^{1}(1-u)^{x} u^{\eta} d u
$$

of which the imaginary part is

$$
\begin{aligned}
\frac{\Gamma(1+x) \Gamma(\eta+1)}{\Gamma(x+\eta+2)} \sin \pi \eta & =-\frac{\Gamma(1+x) \Gamma\left(y-\frac{1}{2} x\right)}{\Gamma\left(1+\frac{1}{2} x+y\right)} \sin \pi\left(y-\frac{1}{2} x\right) \\
& =-\frac{\pi \Gamma(1+x)}{\Gamma\left(1+\frac{1}{2} x+y\right) \Gamma\left(1+\frac{1}{2} x-y\right)} .
\end{aligned}
$$

Consequently (as in [4, (7.149)]), we have for $x \geq 0, y \geq \frac{1}{2} x+1$,

$$
\frac{1}{\pi} \int_{0}^{\pi}\left(2 \cos \frac{1}{2} \theta\right)^{x} \cos y \theta d \theta=\frac{\Gamma(1+x)}{\Gamma\left(1+\frac{1}{2} x+y\right) \Gamma\left(1+\frac{1}{2} x-y\right)} .
$$

By analytic continuation we can conclude that (5) in fact holds for $x>-1$ and all real $y$. Differentiating (5) twice with respect to $y$ and then putting $y=0$ yields

$$
\frac{1}{\pi} \int_{0}^{\pi} \theta^{2}\left(2 \cos \frac{1}{2} \theta\right)^{x} d \theta=2 c(x),
$$

where $-c(x)$ is the coefficient of $y^{2}$ in the power series expansion of the righthand expression in (5). Let

$$
f(y):=\frac{1}{\Gamma\left(1+\frac{1}{2} x+y\right) \Gamma\left(1+\frac{1}{2} x-y\right)} .
$$

Then

$$
f^{\prime}(y)=f(y)\left(\psi\left(1+\frac{1}{2} x-y\right)-\psi\left(1+\frac{1}{2} x+y\right)\right),
$$

so that $f^{\prime}(0)=0$, and hence

$$
f^{\prime \prime}(0)=-2 f(0) \psi^{\prime}\left(1+\frac{1}{2} x\right)=-\frac{2 \psi^{\prime}\left(1+\frac{1}{2} x\right)}{\Gamma^{2}\left(1+\frac{1}{2} x\right)} .
$$

Thus

$$
c(x)=\frac{\Gamma(1+x) \psi^{\prime}\left(1+\frac{1}{2} x\right)}{\Gamma^{2}\left(1+\frac{1}{2} x\right)}=g(x) \psi^{\prime}\left(1+\frac{1}{2} x\right),
$$

where

$$
g(x):=\frac{\Gamma(1+x)}{\Gamma^{2}\left(1+\frac{1}{2} x\right)} .
$$

Differentiating (6) twice with respect to $x$ and then putting $x=0$ yields

$$
\frac{1}{\pi} \int_{0}^{\pi} \theta^{2} \log ^{2}\left(2 \cos \frac{1}{2} \theta\right) d \theta=4 c,
$$


where $c$ is the coefficient of $x^{2}$ in the power series expansion of $c(x)$. Now $g(0)=1$ and

$$
g^{\prime}(x)=g(x)\left(\psi(1+x)-\psi\left(1+\frac{1}{2} x\right)\right),
$$

so that $g^{\prime}(0)=0$, and hence

$$
g^{\prime \prime}(0)=g(0)\left(\psi^{\prime}(1)-\frac{1}{2} \psi^{\prime}(1)\right)=\frac{1}{2} \psi^{\prime}(1)=\frac{\pi^{2}}{12} .
$$

It follows that

$$
c(x)=g(x) \psi^{\prime}\left(1+\frac{1}{2} x\right)=\left(1+\frac{\pi^{2}}{24} x^{2}+\cdots\right)\left(\frac{\pi^{2}}{6}-\zeta(3) x+\frac{\pi^{4}}{120} x^{2}-\cdots\right),
$$

and consequently that

$$
4 c=4\left(\frac{\pi^{4}}{144}+\frac{\pi^{4}}{120}\right)=\frac{11 \pi^{4}}{180} .
$$

Identity (1) follows from (7) and (8).

Proof of the Corollary. First we observe that the ordinary generating function of $H_{n}$ is given by

$$
-\frac{\log (1-z)}{1-z}=\sum_{n=1}^{\infty} H_{n} z^{n}
$$

from which it follows that

$$
\frac{1}{2} \log ^{2}(1-z)=\sum_{n=1}^{\infty} \frac{H_{n}}{n+1} z^{n+1},
$$

with both series converging for $|z|<1$. For $z=r e^{i t}, 0<r<1,0<t \leq \pi$, the imaginary part of the right-hand expression in (9) becomes

$$
\sum_{n=1}^{\infty} \frac{H_{n}}{n+1} r^{n+1} \sin ((n+1) t)
$$

which, by Abel's theorem, tends to

$$
\sum_{n=1}^{\infty} \frac{H_{n}}{n+1} \sin ((n+1) t)
$$

as $r \rightarrow 1-$, the convergence of the latter series being assured because $H_{n} /(n+1)$ tends monotonically to 0 (see [6, Corollary 7.38]). On the other hand, the imaginary part of $\frac{1}{2} \log ^{2}\left(1-r e^{i t}\right)$ tends to $\frac{1}{2}(t-\pi) \log \left(2 \sin \frac{1}{2} t\right)$ as $r \rightarrow 1-$, and hence, for $0<t \leq \pi$,

$$
\frac{1}{2}(t-\pi) \log \left(2 \sin \frac{1}{2} t\right)=\sum_{n=1}^{\infty} \frac{H_{n}}{n+1} \sin ((n+1) t) .
$$

Applying Parseval's theorem to the Fourier series (10), we derive

$$
\frac{1}{2 \pi} \int_{0}^{\pi}(\pi-t)^{2} \log ^{2}\left(2 \sin \frac{1}{2} t\right) d t=\sum_{n=1}^{\infty} \frac{H_{n}^{2}}{(n+1)^{2}} .
$$

On replacing $t$ by $\pi-\theta$ in (11) and using (1) we obtain (2). 
To derive (3) we observe that it follows from (9) that, for $|z|<1$,

$$
\sum_{n=1}^{\infty} \frac{z^{n}}{n^{2}}+\frac{1}{2} \log ^{2}(1-z)=\sum_{n=1}^{\infty} \frac{H_{n}}{n} z^{n}
$$

Equating imaginary parts with $z=e^{i t}$, we obtain as in the derivation of (10) that, for $0<t \leq \pi$,

$$
\sum_{n=1}^{\infty} \frac{\sin n t}{n^{2}}+\frac{1}{2}(t-\pi) \log \left(2 \sin \frac{1}{2} t\right)=\sum_{n=1}^{\infty} \frac{H_{n}}{n} \sin n t .
$$

Now, for $0<r<1,0<t \leq \pi$,

$$
-\int_{0}^{t} \log \left(1-r e^{i u}\right) d u=-i \sum_{n=1}^{\infty} \frac{r^{n}\left(e^{i n t}-1\right)}{n^{2}},
$$

which yields, on letting $r \rightarrow 1-$ and equating real parts,

$$
a(t):=-\int_{0}^{t} \log \left(2 \sin \frac{1}{2} u\right) d u=\sum_{n=1}^{\infty} \frac{\sin n t}{n^{2}} .
$$

This identity, which appears as (4.4) in [3], was numerically investigated by Clausen in 1832, and the function $a(t)$ is Clausen's function $\mathrm{Cl}_{2}(t)$. It follows, by Parseval's theorem, that

$$
\frac{2}{\pi} \int_{0}^{\pi} a(t)^{2} d t=\zeta(4)
$$

and hence, by partial integration,

$$
\frac{2}{\pi} \int_{0}^{\pi} 2 a(t) a^{\prime}(t)(\pi-t) d t=\frac{2}{\pi} \int_{0}^{\pi} a(t)^{2} d t=\zeta(4) .
$$

Applying Parseval's theorem to (12) and using the above identities together with (1), we get

$$
\begin{aligned}
\sum_{n=1}^{\infty} \frac{H_{n}^{2}}{n^{2}} & =\frac{2}{\pi} \int_{0}^{\pi}\left(a(t)+\frac{1}{2} a^{\prime}(t)(\pi-t)\right)^{2} d t \\
& =\zeta(4)+\frac{1}{2} \zeta(4)+\frac{1}{2 \pi} \int_{0}^{\pi}(\pi-t)^{2} \log ^{2}\left(2 \sin \frac{1}{2} t\right) d t \\
& =\frac{3}{2} \zeta(4)+\frac{11}{4} \zeta(4)=\frac{17}{4} \zeta(4),
\end{aligned}
$$

which establishes (3).

Finally, we can deduce (4) from (2) and (3) by observing that

so that

$$
H_{n-1}^{2}=\left(H_{n}-\frac{1}{n}\right)^{2}=H_{n}^{2}+\frac{1}{n^{2}}-\frac{2 H_{n}}{n},
$$

$$
2 \sum_{n=1}^{\infty} \frac{H_{n}}{n^{3}}=\sum_{n=1}^{\infty} \frac{H_{n}^{2}}{n^{2}}+\zeta(4)-\sum_{n=1}^{\infty} \frac{H_{n}^{2}}{(n+1)^{2}}=\frac{5}{2} \zeta(4) .
$$

Identity (4) was discovered by Euler in 1775 , as also was the identity

$$
\sum_{n=1}^{\infty} \frac{H_{n}}{n^{2}}=2 \zeta(3)
$$


In fact, Euler obtained the more general formula

$$
2 \sum_{n=1}^{\infty} \frac{H_{n}}{n^{m}}=(m+2) \zeta(m+1)-\sum_{n=1}^{m-2} \zeta(m-n) \zeta(n+1) \text { for } m=2,3, \ldots
$$

See $[2$, p. 252 , and the references given there] and also, for related material, [7]. Identities such as these involving $H_{n}$ can be derived more easily than those involving $H_{n}^{2}$. They can be dealt with by integrating appropriate generating functions without recourse to Parseval's theorem.

\section{TWO RELATED EXAMPLES}

One may attempt to sum $\left(a_{1}+a_{2}+\cdots+a_{n}\right)^{2} / n^{2}$ for more general sequences. Success depends on being able to get tractable forms of both the generating function (corresponding to (9)) and the Fourier-Parseval integral (corresponding to (11)). If one commences with arctanh instead of log, one is led to

$$
\int_{0}^{\pi} \log ^{2}\left(\tan \frac{1}{4} t\right) d t=\frac{\pi^{3}}{4}
$$

and hence to De Doelder's $[4,(22)]$ formula

$$
\sum_{n=1}^{\infty}\left(\frac{1+\frac{1}{3}+\cdots+\frac{1}{2 n-1}}{n}\right)^{2}=\frac{\pi^{4}}{32}=\frac{45}{16} \zeta(4) .
$$

We also attempted the same process for the sum involving alternating harmonic terms

$$
\sum_{n=1}^{\infty}\left(\frac{1-\frac{1}{2}+\frac{1}{3}-\cdots+\frac{(-1)^{n+1}}{n}}{n}\right)^{2} .
$$

In this case, however, we initially were only able to evaluate the corresponding integral numerically. Moreover, numerical experimentation by David Bailey at NASA Ames Research Center showed that the sum did not have as simple a closed form as in the previous examples, but involved more exotic polylogarithmic constants. In fact his heuristic evaluation of the sum (with vanishingly small probability of error) was

$$
-\frac{13}{8} \zeta(4)+\frac{5}{2} \zeta(2) \log ^{2} 2+\frac{1}{12} \log ^{4} 2+2 \operatorname{Li}_{4}\left(\frac{1}{2}\right),
$$

where $\operatorname{Li}_{4}(x)=\sum_{n=1}^{\infty} \frac{x^{n}}{n^{4}}$. Using arguments involving polylogarithmic and integral identities appearing in [5], we eventually were able to establish Bailey's evaluation rigorously.

5. Modified versions of De Doelder's derivations of (2) AND (13)

In comparing De Doelder's formulae with ours it should be observed that, for $n=1,2, \ldots$,

$$
H_{n}=\psi(n+1)-\psi(1),
$$

and

$$
O_{n}:=\sum_{k=1}^{n} \frac{1}{2 k-1}=\frac{1}{2}\left(\psi\left(n+\frac{1}{2}\right)-\psi\left(\frac{1}{2}\right)\right) \text {. }
$$


Because De Doelder did not choose to highlight these relations, it was not immediately evident from the title of his paper or his abstract that he was dealing with the same series as we were.

Proof of (2). Observe first that

$$
\int_{0}^{1} x^{n} \log ^{m} x d x=(-1)^{m} \frac{m !}{(n+1)^{m+1}} \text { for } n>-1, m=0,1, \ldots,
$$

and hence that

$$
\begin{aligned}
\int_{0}^{1} \frac{\log ^{3}(1-x)}{x} d x & =\int_{0}^{1} \frac{\log ^{3} x}{1-x} d x \\
& =\sum_{n=0}^{\infty} \int_{0}^{1} x^{n} \log ^{3} x d x=-6 \sum_{n=0}^{\infty} \frac{1}{(n+1)^{4}}=-\frac{\pi^{4}}{15} .
\end{aligned}
$$

Next, for $n=0,1, \ldots$,

$$
\begin{aligned}
-\int_{0}^{1} & x^{n} \log (1-x) d x=\int_{0}^{1} x^{n} d x \int_{0}^{x} \frac{1}{1-t} d t \\
\quad= & \int_{0}^{1} \frac{1}{1-t} d t \int_{t}^{1} x^{n} d x \\
\quad= & \frac{1}{n+1} \int_{0}^{1} \frac{1-t^{n+1}}{1-t} d t=\frac{1}{n+1} \int_{0}^{1}\left(1+t+\cdots+t^{n}\right) d t \\
& =\frac{H_{n+1}}{n+1} .
\end{aligned}
$$

It follows from (9), (15), and (16) that

$$
-\frac{1}{2} \int_{0}^{1} \frac{\log ^{3}(1-x)}{x} d x=-\sum_{n=1}^{\infty} \frac{H_{n}}{n+1} \int_{0}^{1} x^{n} \log (1-x) d x=\sum_{n=1}^{\infty} \frac{H_{n} H_{n+1}}{(n+1)^{2}}=\frac{\pi^{4}}{30} \text {, }
$$

and hence, in view of the Euler identity (4), that

$$
\sum_{n=1}^{\infty} \frac{H_{n}^{2}}{(n+1)^{2}}=\sum_{n=1}^{\infty} \frac{H_{n} H_{n+1}}{(n+1)^{2}}-\sum_{n=1}^{\infty} \frac{H_{n}}{(n+1)^{3}}=\frac{\pi^{4}}{30}-\frac{\pi^{4}}{72}+\frac{\pi^{4}}{90}=\frac{11 \pi^{4}}{360} .
$$

Proof of (13). For $0<x<1$, we have

$$
\frac{1}{4}\left(\frac{d}{d x} \log ^{2} \frac{1+x}{1-x}\right)=\frac{\log \frac{1+x}{1-x}}{1-x^{2}}=2 \sum_{n=1}^{\infty} x^{2 n-1} O_{n},
$$

so that

$$
\frac{1}{4} \log ^{2} \frac{1+x}{1-x}=\sum_{n=1}^{\infty} \frac{O_{n}}{n} x^{2 n}
$$

\section{Consequently}

$$
\sum_{n=1}^{\infty} \frac{O_{n}}{n^{2}} x^{2 n}=\frac{1}{2} \int_{0}^{x} \frac{1}{u} \log ^{2} \frac{1+u}{1-u} d u \text { for } 0<x \leq 1 .
$$


Next, it follows from (17) that, for $0 \leq x \leq 1$,

$$
\int_{x}^{1} \frac{1}{u} \log ^{2} \frac{1+u}{1-u} d u=\sum_{n=1}^{\infty} \frac{O_{n}}{n^{2}}\left(1-x^{2 n}\right),
$$

and hence that

$$
\begin{aligned}
\sum_{n=1}^{\infty} \frac{O_{n}^{2}}{n^{2}} & =\sum_{n=1}^{\infty} \frac{O_{n}}{n^{2}} \int_{0}^{1} \frac{1-x^{2 n}}{1-x^{2}} d x=\frac{1}{2} \int_{0}^{1} \frac{d x}{1-x^{2}} \int_{x}^{1} \frac{1}{u} \log ^{2} \frac{1+u}{1-u} d u \\
& =\frac{1}{2} \int_{0}^{1} \frac{1}{u} \log ^{2} \frac{1+u}{1-u} d u \int_{0}^{u} \frac{d x}{1-x^{2}}=\frac{1}{4} \int_{0}^{1} \frac{1}{u} \log ^{3} \frac{1+u}{1-u} d u
\end{aligned}
$$

Substituting $(1-u) /(1+u)=t$ in the final integral and then applying (14), we obtain

$$
\begin{aligned}
\sum_{n=1}^{\infty} \frac{O_{n}^{2}}{n^{2}} & =-\frac{1}{2} \int_{0}^{1} \frac{\log ^{3} t}{1-t^{2}} d t=-\frac{1}{2} \sum_{n=0}^{\infty} \int_{0}^{1} t^{2 n} \log ^{3} t d t \\
& =\sum_{n=0}^{\infty} \frac{3}{(2 n+1)^{4}}=3 \zeta(4)-\frac{3}{16} \zeta(4)=\frac{\pi^{4}}{32}
\end{aligned}
$$

This establishes (13), which is the corrected version of identity (22) in [4]. As a bonus we also obtain from (17), on substituting $x=1$ and $(1-u) /(1+u)=t$, and then applying (14), that

$$
\begin{aligned}
\sum_{n=1}^{\infty} \frac{O_{n}}{n^{2}} & =\int_{0}^{1} \frac{\log ^{2} t}{1-t^{2}} d t=\sum_{n=0}^{\infty} \int_{0}^{1} t^{2 n} \log ^{2} t d t \\
& =\sum_{n=0}^{\infty} \frac{2}{(2 n+1)^{3}}=\frac{7}{4} \zeta(3),
\end{aligned}
$$

which is identity (15) in [4].

It should be noted that De Doelder's derivation of (2) in conjunction with (11) provides an alternative proof of (1).

\section{REFERENCES}

1. T. M. A. Apostol, Mathematical analysis, Second edition, Addison-Wesley, Cambridge, MA, 1975.

2. B. C. Berndt, Ramanujan's notebooks, Part I, Springer-Verlag, New York, 1985.

3. R. B. Burckel, An introduction to classical complex analysis, Volume 1, Academic Press, New York, 1979.

4. P. J. De Doelder, On some series containing $\psi(x)-\psi(y)$ and $(\psi(x)-\psi(y))^{2}$ for certain values of $x$ and $y$, J. Comput. Appl. Math. 37 (1991), 125-141.

5. L. Lewin, Polylogarithms and associated functions, North-Holland, New York, 1981.

6. K. R. Stromberg, An introduction to classical real analysis, Wadsworth, Belmont, CA, 1981.

7. A. J. van der Poorten, Some wonderful formulae...An introduction to polylogarithms, Proceedings of Number Theory Conference, Kingston, Ontario, 1979, pp. 269-286.

Department of Mathematics, University of Western Ontario, London, Ontario, CANADA N6A 5B7

Department of Mathematics and Statistics, Simon Fraser University, Burnaby, British Columbia, Canada V5A IS6 